\title{
Metodología de desarrollo de un procedimiento para la resolución de conflictos y acoso psicológico laboral
}

\author{
Development of a procedure for the resolution of \\ conflict situations and psychological harassment at work
}

\author{
Antonia Bernat Jiménez \\ Hospital Clínico de San Carlos. Madrid. España. \\ Covadonga Caso Pita \\ Hospital Clínico de San Carlos. Madrid. España. \\ Recibido: 18-05-10 \\ Aceptado: $11-0610$ \\ Correspondencia: \\ Antonia Bernat Jiménez \\ S. ${ }^{\circ}$ PRL. Área Psicosociológica \\ Hospital Clínico San Carlos \\ Martín Lagos, s/n \\ 28040 Madrid. España. \\ Tfno: 913303431 \\ e-mail: abernat.hcsc@salud.madrid.org
}

Resumen

El artículo se centra en la descripción de la metodología utilizada en la elaboración de un procedimiento de resolución de conflictos y acoso psicológico en el entorno laboral.

El procedimiento se encuadra dentro de una línea de trabajo de diseño preventivo, que pretende constituir un programa de actuación para prevenir la violencia interna en una organización sanitaria.

La característica fundamental de este desarrollo, es apostar por la corresponsabilidad de todos los miembros de la organización a la hora de elaborar e implementar las herramientas que constituyen el programa preventivo.

Med Segur Trab (Internet) 2010; 56 (219): 124-131

Palabras clave: Violencia en el lugar de trabajo, prevención riesgos psicosociales, conflictos, acoso psicológico, personal sanitario.

Abstract

This article is focused on describing the methodology used in the creation of a conflict situation and psychological harassment at work resolution procedure.

The described procedure is part of a preventive design line of work intended to constitute an action plan to prevent violence within a healthcare organization.

Its main feature is the importance given to the co-responsability of every member of the organization in terms of creating and implementing the preventive measures that constitute the preventive program itself.

Med Segur Trab (Internet) 2010; 56 (219): 124-131

Key Words: Workplace violence, psychosocial risks prevention, conflicts, psychological harassment, healthcare personnel. 
Dentro de una línea de diseño preventivo de los riesgos psicosociales, concretamente aquellos que tienen su origen en las relaciones interpersonales, el presente trabajo aborda la metodología utilizada en el desarrollo de una serie de instrumentos (específicamente el procedimiento para la resolución de conflictos y acoso psicológico en el trabajo), que buscan un cambio planeado, centrado, en este caso, en la mejora de las relaciones humanas y la prevención de la violencia en el lugar de trabajo.

Con esta intervención se busca conjugar planteamientos preventivos y de diseño organizacional, esperando lograr, al intervenir sobre actitudes, relaciones y clima laboral, que el cambio pase a formar parte de los valores y la cultura de la organización, repercutiendo tanto en la satisfacción y la salud de los profesionales como en la calidad asistencial.

El objetivo principal de este artículo es describir la metodología utilizada en la elaboración de un procedimiento para el abordaje y la resolución de conflictos y acoso psicológico en el trabajo en el ámbito sanitario. La necesidad de este instrumento se constató por la observación, desde las diferentes instancias implicadas (dirección, mandos intermedios, servicio de Prevención de Riesgos Laborales y delegados de prevención), de la existencia de una serie de conflictos interpersonales que estaban afectando al rendimiento y la satisfacción de los trabajadores.

Dentro de esta línea preventiva, la primera actuación que se abordó, siguiendo la misma metodología que la que se describe para el actual procedimiento, fue la definición de un marco de regulación de las relaciones dentro de la Organización (Código de conducta), basado en el respeto mutuo y la mejora de la comunicación, cuya finalidad es la prevención de comportamientos o actitudes inadecuadas en el lugar de trabajo.

Conscientes de que el recurso humano es decisivo en cualquier organización y de la importancia que los riesgos psicosociales alcanzan en el medio sanitario, estas herramientas, código de conducta y procedimiento que se presentan, se ven como instrumentos que pueden actuar sobre el desarrollo de relaciones humanas que permitan prevenir los conflictos, así como abordarlos rápida y oportunamente cuando se tengan indicios de su aparición.

\section{INTRODUCCIÓN}

La actividad sanitaria se caracteriza por un sistema de relaciones interpersonales complejo, con frecuentes interacciones, no solo con usuarios, sino entre diferentes profesionales, muchas veces con objetivos y plazos temporales distintos.

Una consecuencia de no saber afrontar adecuadamente estas relaciones interpersonales tan diversas son los conflictos, cuyas causas pueden ser múltiples, formando parte, algunas de ellas, de las propias condiciones de trabajo: déficits organizativos, de liderazgo, una excesiva competitividad, falta de reconocimiento, etc.

Hay que tener en cuenta que todo conflicto está siempre presidido por alguna de las innumerables expresiones de la violencia, por lo que, tanto los conflictos como el acoso psicológico, constituyen un riesgo psicosocial.

El abordaje temprano de los conflictos y el desarrollo de estrategias para la resolución de los mismos, contribuyen a una mejora del clima y la salud laboral, así como a la identificación y control de factores de riesgo relacionados con la violencia psicológica en el lugar de trabajo.

La diversidad de causas que generan conflicto obligan a plantear las intervenciones de manera plural y abierta, con procedimientos flexibles, que se centren en el entorno de trabajo, además de en las personas implicadas en el conflicto. El éxito de estas actuaciones requiere del compromiso de todos los miembros de la organización, cada uno en su responsabilidad. 


\section{DEFINICIONES}

- Las relaciones personales como factor de riesgo psicosocial:

Las personas tienen una serie de necesidades sociales que son fuente de motivación, por ello, las relaciones en el puesto de trabajo pueden ser fuente de satisfacción, en cuyo caso contribuyen a disminuir el estrés o a amortiguar sus consecuencias, pero cuando estas relaciones son inadecuadas, pueden constituir por si mismas una importante fuente de estrés.

- El conflicto podría definirse como una situación de desacuerdo entre dos o más personas, en la que cada una de las partes tiene intereses o posiciones contrapuestas.

Cuando estas percepciones divergentes se hacen incompatibles, el conflicto se consolida en su vertiente negativa, manifestándose en incomunicación o comunicación violenta, afectando al clima organizacional y a la salud laboral e incidiendo negativamente en el desarrollo del trabajo.

- El acoso psicológico laboral se puede definir como la situación en que un trabajador o grupo de trabajadores ejercen una violencia psicológica extrema sobre otro trabajador o trabajadores, de forma sistemática y recurrente, durante un tiempo prolongado. La conducta objetiva, percibida como ataque, se ejerce en el trabajo, mediante maniobras como limitar la comunicación y el contacto social de la víctima, desprestigiar a la persona, desacreditar su capacidad profesional y comprometer su salud psíquica, con la finalidad de conseguir el abandono del lugar de trabajo.

\section{PROCEDIMIENTO PARA LA RESOLUCIÓN DE LAS SITUACIONES DE CONFLICTO Y ACOSO PSICOLÓGICO EN EL ENTORNO LABORAL}

El procedimiento establece las actuaciones a realizar en materia de prevención, resolución y seguimiento de las situaciones de conflicto y acoso psicológico en el entorno laboral.

\section{Justificación y método}

La mejora de las relaciones humanas y específicamente la prevención de la violencia en el lugar de trabajo, se encuadra dentro de las líneas estratégicas de la organización en materia de prevención y desarrollo organizacional.

Desde el Servicio de Prevención, donde se llevaba largo tiempo trabajando en esta materia, se partía de unas premisas:

- La larga experiencia del área de Psicosociología en la intervención en este tipo de riesgos

- El conocimiento profundo del medio (a través de la experiencia profesional, los datos procedentes de evaluaciones psicosociales, análisis de clima laboral, etc.)

Esta situación evidenciaba que cualquier intervención que pretendiera lograr un consenso importante, una amplia difusión y una implantación efectiva, iba a requerir del compromiso de todos los miembros de la organización, cada uno en sus responsabilidades, empezando por la dirección del Centro.

Así, surge la convicción de que cualquier diseño preventivo, en relación con el riesgo psicosocial, que pretenda ser integral y efectivo, debería ser no solo sancionado, sino elaborado, de forma participativa, en el seno del máximo órgano competente en materia de prevención laboral dentro de la organización, el Comité de Seguridad y Salud.

En línea con este planteamiento, desde el Servicio de Prevención de Riesgos Laborales se decide adquirir un papel activo, impulsando la creación, a efectos de una mayor 
eficacia, de un grupo de trabajo permanente, Grupo de Psicosociales, derivado del Comité de Seguridad y Salud, dedicado al análisis, la definición y elaboración de actuaciones dirigidas a la prevención del riesgo psicosocial en la organización.

\section{Composición del grupo de trabajo de psicosociales}

En la composición del grupo de trabajo, cara al consenso y a la participación, se mantiene la representación tanto de la administración como de la parte social (dirección y delegados de prevención), incluyendo a dos miembros del Servicio de Prevención, representantes de las áreas de Vigilancia de la Salud y Psicosociología, coordinando el grupo este último.

\section{Actuaciones}

Se acuerda que, en esta primera fase, todas las actuaciones se centrarán en la mejora de las relaciones interpersonales en el lugar de trabajo.

Tras elaborar el Código de conducta, que establece un marco general de regulación de las relaciones en la organización, basadas en el respeto mutuo y la mejora de la comunicación, se aborda desde el grupo el siguiente instrumento preventivo del programa: el Procedimiento para la Resolución de Situaciones de Conflicto y Acoso Psicológico, cuya metodología de elaboración presentamos.

\section{Forma de trabajo}

- Convocatorias:

- Periodicidad mensual

- En cada reunión se pacta la fecha de la siguiente y la coordinación envía convocatoria por correo electrónico

- Reuniones extraordinarias según necesidades

- Se proponen fechas desde la Coordinación

- Orden del día

- Los contenidos de cada reunión se acuerdan en la previa

- Se trabaja a partir de las propuestas técnicas del área de Psicosociología, que se remiten a todos los miembros, en tiempo y forma, a través del correo electrónico

\section{Contenido de sesiones}

- Identificación de problemas

- Análisis de la situación

- A partir de la información disponible y desde las diferentes perspectivas existentes en el grupo: dirección, parte social y Servicio de Prevención

- Estrategias de intervención que se venían utilizando

- Selección de nueva estrategia y Objetivos

Diseño preventivo $\rightarrow$ Desarrollo del procedimiento de resolución de conflictos y acoso psicológico en el trabajo

- Estudio de propuestas técnicas:

- Debate de las propuestas técnicas elaboradas y remitidas a los miembros del grupo por la coordinación, representante del área de Psicosociología del Servicio de Prevención. Aportaciones y consenso.

- La coordinación realiza las modificaciones acordadas en cada reunión y vuelve a remitir el documento, por correo electrónico, a todos los miembros. 


\section{Aprobación del procedimiento}

- El documento final del grupo de trabajo, se presenta y sanciona en Comité de Seguridad y Salud

- El hecho de que llegue consensuado por el grupo de trabajo, con representación similar a la existente en el Comité, asegura su aprobación de forma inmediata en el mismo

\section{Difusión e implantación}

- El procedimiento, sancionado por el Comité, vuelve al grupo de trabajo de Psicosociales, que pone en marcha las medidas, ya establecidas por consenso, para llevar a cabo la difusión de la herramienta aprobada:

- Elaboración de díptico para difusión entre todos los profesionales

- Colgar en Intranet procedimiento y díptico

- Artículo informativo sobre el procedimiento en la revista de la organización

- Envío de correo masivo institucional, a todos los trabajadores que dispongan de dirección, comunicando la puesta en marcha del procedimiento y las fuentes de información

- Carta de la dirección a todos los responsables de personal informando del acceso a la información y solicitando su implicación en la difusión e implantación del procedimiento

- Desde el momento de su aprobación e inicio de la difusión, la herramienta se implanta en la organización, por tanto, todas las instancias implicadas (dirección, delegados de prevención y Servicio de Prevención), asumirán las nuevas actuaciones previstas en el procedimiento, informando de las mismas a los trabajadores que deberán también secundarlas.

\section{Resumen del contenido y actuaciones contempladas por el procedimiento}

\section{Garantías}

- El procedimiento se llevará a cabo en un plazo máximo de 30 días

- Se seguirá en todo momento el principio de confidencialidad

- No represalias

Fases:

- Fase de detección del conflicto e inicio del procedimiento mediante presentación de queja

Tras presentación de la Queja, la Subdirección de Recursos Humanos junto con el área de Psicosociología del Servicio de Prevención, valorarán la intervención y su responsable, según el tipo de conflicto (gradación de la violencia) y la implicación del mando en el mismo.

- Fase de intervención

- Información y evaluación

- Análisis del problema

- Resolución e informe

- Implantación de medidas y seguimiento

\section{- Derivación a instancia superior}

Tendrá lugar en caso de que el mando intermedio apareciera como implicado en el conflicto o de que el mismo no se de por resuelto en el nivel que ha realizado la intervención. 


\section{- Intervención del Servicio de Prevención}

El Servicio de Prevención, que permanecerá informado a través de la Subdirección de Recursos Humanos del estado de la situación durante todo el proceso, podrá prestar apoyo especializado a los responsables en cualquiera de las fases indicadas y actuará en primera instancia en aquellos casos en que, por el tipo de problema (altos niveles de violencia o afectación personal o del grupo...), así se decida en la fase de "información y evaluación".

Igualmente actuará en los casos en que, tras la intervención de los niveles jerárquicos contemplados, no se haya alcanzado una solución al problema.

Como funciones asignadas, se llevarán a cabo todas las actuaciones oportunas para identificar los factores de riesgo presentes en las condiciones de trabajo, así como para garantizar la seguridad y la salud de los trabajadores.

Las actuaciones del Servicio de Prevención seguirán, en lo esencial, las fases de intervención ya propuestas, incluyendo las funciones específicas indicadas para cada caso:

- Mediación

- Evaluación de condiciones de trabajo y establecimiento de medidas preventivas

- Intervención médica y psicológica sobre el trabajador si procede, etc.

El Servicio de Prevención podrá así mismo proponer, si la gravedad del conflicto lo requiere, medidas preventivas/cautelares, ante la Subdirección de Gestión de Recursos Humanos y las Direcciones competentes, en relación con las personas que intervienen en el proceso.

\section{Registro de datos}

Se crea un registro de datos de intervenciones, del que a efectos de guarda y confidencialidad de los mismos, se responsabiliza el Servicio de Prevención.

Se harán constar los datos relativos al incidente, así como las propuestas, estableciéndose un seguimiento periódico de los resultados.

A partir de este registro centralizado, se llevará a cabo la explotación periódica de los datos, a fin de informar, sobre los datos pactados que garantizan la confidencialidad, al Comité de Seguridad y Salud, analizar el mapa de riesgos y establecer las medidas preventivas pertinentes.

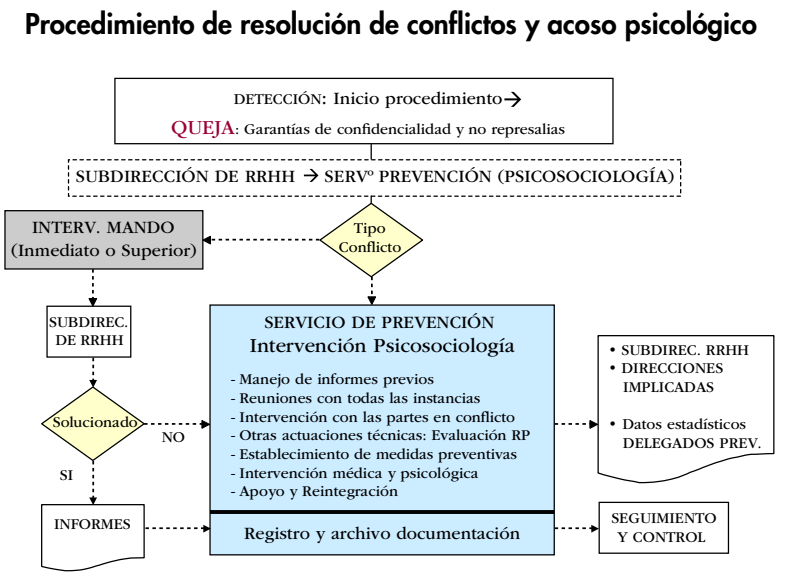




\section{CONCLUSIONES}

En la actualidad, un aspecto vital a considerar en cualquier organización es el abordaje de la violencia.

En el ámbito laboral es la violencia psicológica la que se da con mayor frecuencia, siendo el sector de la salud uno de los más vulnerables a este tipo de agresiones, que pueden presentarse de varias formas: abuso verbal, amenazas, acoso...

Entre los diferentes tipos de violencia que pueden darse en el lugar de trabajo, el artículo se ha centrado en la violencia interna, es decir, la que se da entre los profesionales de la organización y cuyas causas pueden ser múltiples: factores sociales, laborales y personales.

La violencia interna en las organizaciones sanitarias, afecta a la satisfacción y la salud de los trabajadores, repercutiendo en el clima organizacional, la productividad y la calidad asistencial. Más allá de una mera toma de conciencia del problema, es imperativo analizarlo e incluir acciones en el medio laboral para prevenir este riesgo.

Hoy en día tanto desde la Prevención del riesgo psicosocial, como desde el desarrollo organizacional, centrado en los recursos humanos, se elaboran y proponen diferentes programas tendentes a reducir la violencia en los lugares de trabajo. No obstante, existe una carencia de investigaciones rigurosas que empleen diseños adecuados para medir su impacto.

En el diseño del programa que se presenta se consideró, en primer lugar, el tipo de violencia al que iba destinada la prevención, así como las necesidades específicas de intervención y las características de la organización. Es evidente, que no se debe asumir, para un determinado programa, su igual funcionamiento en cualquier organización y bajo cualquier circunstancia.

Actuaciones preventivas incluidas en el programa y puestas en marcha hasta la fecha:

- Declaración, por parte de la Gerencia, de tolerancia cero a la violencia, dentro del Plan de Prevención de la organización

- Código de conducta: regulación de las relaciones personales

- Formación en prevención de riesgos psicosociales

- Estímulo a la comunicación de conductas violentas (quejas)

- Procedimiento para la resolución de conflictos y acoso psicológico

Actuaciones que se proponen incluir a futuro:

- Formación, a los responsables de personal, en manejo de conflictos (conscientes de la influencia del estilo de liderazgo en la gestión de los mismos).

- Mejora de las condiciones de trabajo: que pueden favorecer la aparición y mantenimiento de conductas no deseadas.

La línea de trabajo presentada está desarrollada, de forma participada, para su aplicación en la prevención de la violencia psicológica de origen interno, en una organización específica, con unos medios y necesidades también específicos, siendo uno de los objetivos del programa el que estas buenas prácticas lleguen a formar parte, a medio o largo plazo, de los valores y la cultura de la organización.

\section{AGRADECIMIENTOS}

Los autores, componentes del Servicio de Prevención de Riesgos Laborales de la organización, agradecen a todos los que han participado en el desarrollo de las herramientas preventivas presentadas, su implicación y colaboración:

- Dirección del Centro, Delegados de Prevención, resto de componentes del Comité de Seguridad y Salud y, fundamentalmente, a todos los miembros del grupo de trabajo de Psicosociales, por su empeño y voluntad de consenso. 


\section{REFERENCIAS BIBLIOGRÁFICAS}

Ancland, A. F., Cómo utilizar la mediación para resolver conflictos en las organizaciones, Paidós, Barcelona, 1993

Bennett, J. and W. Lehman, the relationship between problem, co-workers and quality work practices. Work and Stress, 1999. 13(4): p. 299-311.

Burton, J. Conflict: Resolution and Provention. London. Macmillan, 1990.

Burgess, H \& G. Encyclopedia of Conflict Resolution. Santa Bárbara, California: ABC-CLIO.

Daus, C.S., Bullying and emocional abuse in the workplace: internacional Perspectives in Research and Practice. Personnel Psychology, 2004. 57(4): p. 1088-1091.

Comunidad Foral de Navarra, Procedimiento en materia de prevención, resolución y seguimiento de las situaciones de conflicto y acoso psicológico en el entorno laboral.

Giraudier, M., Cómo gestionar el clima laboral. Ediciones Obelisco, 2004. p. 69-74.

Martín F, Pérez J, López JA. Nota Técnica de Prevención 476: El hostigamiento psicológico en el trabajo: Mobbing. Instituto Nacional de Seguridad e Higiene en el Trabajo; 1998.

Mitchell, C.; Banks, M. Handbook of Conflict Resolution. The analytical problem solving approach. Londres: Pinter, 1996.

Osakidetza, Protocolo de actuación. Violencia en el trabajo.

INSHT, NTP 489: Violencia en el lugar de trabajo.

Peiró, J.M. y Prieto, F., Tratado de Psicología del Trabajo. V. II Aspectos psicosociales del trabajo. Madrid. Síntesis, 1996.

Preventing measures may reduce workplace violence. Nation's Health, 2001. 31(4): p.7.

Universidad Nacional de Educación a Distancia, Protocolo de actuación en materia de acoso.

Vinyamata, E. Manual de prevención y Resolución de conflictos. Barcelona: Ariel, 1999.

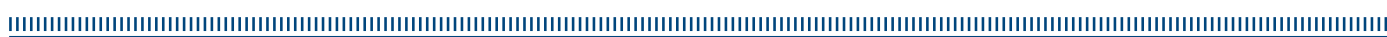

